\title{
Metastatic Inhibitory Effect
}

National Cancer Institute

\section{Source}

National Cancer Institute. Metastatic Inhibitory Effect. NCI Thesaurus. Code C45983.

Metastatic inhibitory effect results in the interference or prevention of cellular neoplastic transformation. Several therapeutic agents can be utilized to this end, such as drugs and monoclonal antibodies. 